\title{
Relativity and the Solar Wind: The Maxwell-Equation Origins of the Solar-Wind Motional Electric Field
}

\author{
Joseph E. Borovsky ${ }^{1,2}$ \\ ${ }^{1}$ Space Science Institute, Boulder, CO, USA \\ ${ }^{2}$ CSSE, University of Michigan, Ann Arbor, MI, USA \\ Email: jborovsky@spacescience.org
}

Received 12 May 2016; accepted 20 August 2016; published 23 August 2016

Copyright (C) 2016 by author and Scientific Research Publishing Inc.

This work is licensed under the Creative Commons Attribution International License (CC BY).

http://creativecommons.org/licenses/by/4.0/

(c) (i) Open Access

\section{Abstract}

The motional electric field of the solar wind as seen by the Earth is examined theoretically and with spacecraft measurements. As it flows outward from the sun, the solar-wind plasma carries a spatially structured magnetic field with it. To calculate the motional electric field of the solar wind the spatially structured magnetic field is Lorentz transformed; for a full physical understanding, it is also necessary to Lorentz transform the current densities and charge densities in the solar wind. Referring to Maxwell's equations, two related questions are asked: 1) Is the source of the solarwind motional electric field charge density in the solar wind, time derivatives of current densities in the solar wind, or both? 2) Is the solar-wind motional electric field at Earth an electrostatic field, an induction field, or a superposition of the two? A Helmholtz decomposition of the motional electric field of the solar wind is made into a divergence-origin (electrostatic) and a curl-origin (induction) electric field. The global electric field associated with the outward advection of the global Parker-spiral magnetic field is found to be electrostatic with its origin being a distributed charge density in the solar-wind plasma. The electrostatic versus induction nature of the time-varying electric field associated with the advection of mesoscale magnetic structure varies with time as differently shaped magnetic structures in the solar-wind plasma pass the Earth; the mesoscale structure of the solar-wind plasma contains sheets of space charge and sheets wherein the current density has nonzero time derivatives.

\section{Keywords}

Lorentz Transformation, Electric Field, Induction, Helmholtz Decomposition, Solar Wind, Magnetic Structure, Heliosphere, Plasma 


\section{Introduction}

The solar wind is a magnetized collisionless plasma comprised of ions (mostly protons) and electrons. (Collisionless means that inter-particle collisions that would disrupt the ion or electron orbits in the magnetic and electric fields are rare.) The solar-wind plasma flows at a high velocity radially outward from the Sun, in the range $250 \mathrm{~km} / \mathrm{s}$ to $1000 \mathrm{~km} / \mathrm{s}$. Typical parameters for the solar wind at Earth appear in Table 1 . The magnetic field $\underline{B}$ in the solar-wind plasma originates at the Sun and is carried outward with the supersonic plasma flow. Because of the rotation of the Sun and the radial outflow of the wind, the magnetic field in the solar wind has on average a "Parker-spiral" orientation [1]. The magnetic-field vector has strong localized variations about the Parker-spiral direction: these spatial variations in $\underline{B}$ are advected outward with the solar-wind flow [2]. Much of the spatial variation of $\underline{B}$ is owed to the presence of current sheets embedded in the solar-wind plasma [3] [4], with the value of the magnetic-field vector $B$ jumping across the current sheets.

The motional electric field $\underline{E}$ of a plasma is a Lorentz transformation of the magnetic field $\underline{B}$ in the plasma [5]. For a velocity vector $\underline{v}$ between two reference frames, the Lorentz transformation of the components of the electric and magnetic fields parallel to and perpendicular to $\underline{\underline{v}}$ is $[6]$

$$
\begin{gathered}
E_{\|}=E_{\|}^{\prime} \\
E_{\perp}=E_{\perp}^{\prime}-(\gamma / c) \underline{v} \times \underline{B}^{\prime} \\
B_{\|}=B_{\|}^{\prime} \\
B_{\perp}=B_{\perp}^{\prime}+(\gamma / c) \underline{v} \times \underline{E}^{\prime}
\end{gathered}
$$

where $\gamma=\left(1-(v / c)^{2}\right)^{-1 / 2}$ and where we will take the primed frame to be at rest in the solar-wind plasma and the unprimed frame to be the rest frame of the Earth or the Sun. In the rest frame of the solar-wind plasma, $E_{\perp}^{\prime}=0$. This is because (in the absence of non-electromagnetic forces such as gravity) in a collisionless plasma particle orbit theory says that the ions and the electrons E-cross-B drift with an averaged velocity of $\underline{v}_{d r i f t}=c\left(\underline{E}^{\prime} \times \underline{B}^{\prime}\right) /\left|\underline{B}^{\prime 2}\right|$ (cf. eq. (12.43) of Reference [6]). In the rest frame of the plasma there can be no E-cross-B drift velocity of the ions and electrons, otherwise it would not be the rest frame of the plasma. Therefore, in the rest frame of the solar-wind plasma $E_{\perp}^{\prime} \equiv 0$. In the reference frame of the Earth or the Sun, the plasma flow is of the order of $\mathrm{v} / \mathrm{c} \sim 10^{-3}$, so $\gamma \cong 1$ can be taken. For $E_{\perp}^{\prime}=0$ expression (1d) gives $B_{\perp}=B_{\perp}^{\prime}$. For $\gamma \cong 1, E_{\perp}^{\prime}=0$, and $B_{\perp}^{\prime}=B_{\perp}$, expression (1b) yields the motional electric field $\underline{E}$ for a plasma moving with a velocity $\underline{\mathrm{v}}$ in a magnetic field $\underline{\mathrm{B}}$ to be

$$
\underline{E}=-(1 / c) \underline{v} \times \underline{B}
$$

which is the low-velocity Lorentz transformation common for a moving conducting fluid [6] [7]. The solar-wind motional electric field as seen by the Earth (expression (2)) is often taken to be the driver of geomagnetic activity in the Earth's magnetosphere-ionosphere system [8] (but see Reference [9] for a contrary interpretation).

In this paper, we wish to gain an understanding of the solar-wind motional electric field from first principles. The motional electric field of the solar wind will be examined via the Lorentz transformation of the magnetic-field structure in the solar wind. Because of the spatial variations of the magnetic-field vector $\underline{B}$, the motional electric field will have spatial variations. Via Maxwell's equations, the sources of the motional electric field will be sought: charge densities in the solar wind and time derivatives of current densities in the solar wind. The question will be asked whether the motional electric field of the solar wind is an electrostatic electric field, an

Table 1. Typical values (mean values) of solar-wind parameters at Earth.

\begin{tabular}{ccc}
\hline Symbol & Mean Value & Definition \\
\hline$v_{s w}$ & $438 \mathrm{~km} / \mathrm{s}$ & solar-wind speed \\
$|\underline{B}|$ & $6.3 \times 10^{-5} \mathrm{Gauss}$ & magnetic-field strength \\
$n$ & $6.8 \mathrm{~cm}^{-3}$ & proton number density \\
$T_{p}$ & $1.1 \times 10^{5} \mathrm{~K}$ & proton temperature \\
$M$ & 5.9 & magnetosonic Mach number
\end{tabular}


inductive electric field, or both. We wish to gain an understanding of the solar-wind motional electric field from first principles. Identifying the electrostatic versus induction portions of electric fields within the Earth's magnetosphere has been of interest because the identification yields an understanding of the origin of the electric fields and a capability to add them to numerical models of the magnetosphere [10] [11].

To avoid confusion, the reader should note that there is also a non-motional electric field in the solar wind associated with the "interplanetary electrostatic potential" or "heliospheric electric potential" [12]. This is a global parallel-to- $\underline{B}$ electric field associated with the differences in the collisionless transport of the solar-wind electrons and the solar-wind ions along the magnetic field. The sign of this interplanetary electric field is outward along the magnetic field lines, accelerating ions away from the Sun and retarding the outward electron flux. This interplanetary electrostatic potential is responsible for the observed statistical increase of the bulk speed of the solar wind $v_{s w}$ between $0.3 \mathrm{AU}$ and $1 \mathrm{AU}$. This interplanetary electrostatic potential is not the subject of the present study.

\section{Electrostatic Fields versus Induction Fields and Charge Density versus Time-Varying Current Density}

According to the Helmholtz decomposition [13] [14], any vector field can be decomposed into the sum of an irrotational (curl-free) field plus a solenoidal (divergence-free) field. Hence, any vector electric field $\underline{E}$ can be written as the superposition

$$
\underline{E}=\underline{E}_{e s}+\underline{E}_{i n d}
$$

where $\underline{E}_{e s}$ is an electrostatic electric field that is everywhere curl-free and $\underline{E}_{\text {ind }}$ is an induction electric field that is everywhere divergence-free. Further, the field $\underline{E}$ can be determined everywhere in space from knowledge of the values of the divergence of the field $\underline{E}$ and the curl of the field $\underline{E}$ everywhere in space [14]. This determination of $\underline{E}$ from $\nabla \cdot \underline{E}$ and $\nabla \times \underline{E}$ is simplified by the decomposition as follows. When one takes $\nabla \cdot \underline{E}$ one finds the regions where $\nabla \cdot \underline{E}_{e s} \neq 0$ and when one takes $\nabla \times \underline{E}$ one finds the regions, where $\nabla \times \underline{E}_{\text {ind }} \neq 0$. From all of the regions of $\nabla \cdot \underline{E}_{e s} \neq 0$ one can obtain $\underline{E}_{e s}$ everywhere in space and from all of the regions where $\nabla \times \underline{E}_{i n d} \neq 0$ one can obtain $\underline{E}_{\text {ind }}$ everywhere in space. Knowing $\underline{E}_{e s}$ everywhere in space and $\underline{E}_{\text {ind }}$ everywhere in space, one can obtain $\underline{E}$ everywhere in space.

For this paper the following equivalence of adjectives for each of the two types of electric fields is declared. First type: inductive $=$ divergence-free $=$ solenoidal $=$ non-potential $=$ transverse. Second type: electrostatic $=$ curl-free $=$ irrotational $=$ potential $=$ longitudinal. For an electrostatic field $\underline{E}_{e s}$, the electric-field lines end; for an induction field $\underline{E}_{\text {ind }}$, the electric-field lines do not end.

Here we will define a purely electrostatic electric field $\underline{E}_{e s}$ as an irrotational field having its origin in regions where the divergence $\nabla \cdot \underline{E}$ is nonzero. Likewise, we will define a purely induction electric field $\underline{E}_{\text {ind }}$ as a solenoidal field having its origin in regions where the curl $\nabla \times \underline{E}$ is nonzero. From this and from expression (3) it follows that $\nabla \cdot \underline{E}=\nabla \cdot \underline{E}_{e s}$ and that $\nabla \times \underline{E}=\nabla \times \underline{E}_{\text {ind }}$. From Coulomb's law

$$
\nabla \cdot \underline{E}_{e s}=4 \pi \rho_{q},
$$

it is seen that the electrostatic field originates from regions of nonzero charge density $\rho_{q}$. From Faraday's law of induction

$$
\nabla \times \underline{E}_{\text {ind }}=-(1 / c) \partial \underline{B} / \partial t,
$$

it is seen that the induction field originates from regions of nonzero $\partial \underline{B} / \partial t$. It is important to explore expression (5) a little further. Taking the curl of Faraday's law $\nabla \times \nabla \times \underline{E}_{\text {ind }}=-(1 / c) \partial / \partial t(\nabla \times \underline{B})$, using Ampere's law $\nabla \times \underline{B}=(4 \pi / c) \underline{j}$ with the displacement current neglected (negligible in the $v \ll c$ limit [15]) to replace $\nabla \times \underline{B}$, and taking $\nabla \cdot \underline{E}_{\text {ind }}=0$ in the expansion $\nabla \times \nabla \times \underline{E}_{\text {ind }}=\nabla^{2} \underline{E}_{\text {ind }}-\nabla\left(\nabla \cdot \underline{E}_{\text {ind }}\right)$ yields

$$
\nabla^{2} \underline{E}_{\text {ind }}=\left(4 \pi / c^{2}\right) \partial \underline{j} / \partial t
$$

(with $\nabla \cdot \underline{E}_{i n d}=0$ ) for the induction field, which indicates that the induction electric field $\underline{E}_{\text {ind }}$ has its origin in regions where the time derivative of the current density $\partial j / \partial t$ is nonzero.

The motional electric field $\underline{E}=-(1 / c) \underline{v} \times \underline{B}$ of the solar wind is sometimes called the induced or inductive 
electric field of the solar wind because it is "induced" by the motion of the plasma. In this paper more care will be taken with the "induction" nomenclature.

\section{Observations of the Solar-Wind Motional Electric Field at Earth}

In Figure 1, the solar-wind speed and two components transverse to radial (from the Sun) of solar-wind motional electric field near Earth are plotted as functions of time for 100 days in the year 2004. The GSE (Geocentric Solar Ecliptic) right-handed coordinate system is used where X points from the Earth toward the Sun, Y points transverse to the Sun-Earth line in the ecliptic plane opposite to the direction of the Earth's motion about the Sun, and $\mathrm{Z}$ is normal to the ecliptic plane pointing in the northward direction. In the top panel the solar-wind radial velocity $v_{s w}$ is plotted, in the middle panel and bottom panel the north-south component $E_{z}$ and the dawn-dusk component $E_{y}$ of the solar-wind motional electric field $\underline{E}=-(1 / c) \underline{v}_{s w} \times \underline{B}$ are plotted. The black points are 64-s time resolution values and the red points are 24-hr running averages. The electric field is calculated from measurements of the bulk-flow vector $\underline{v}_{s w}$ by the SWEPAM instrument [16] on the ACE spacecraft in the solar wind and from measurements of the magnetic-field vector $\underline{B}$ from the MAG instrument [17] on ACE. The value of the observed motional electric field depends on the reference frame of the observer, in this case the ACE spacecraft in approximately the rest frame of the Earth. Note the reversals in the sign of the north-south component $E_{z}$ (e.g. Days 111, 122, 139, 149, 165, 177, and 191): these reversals occur at sector reversals of the solar-wind magnetic field wherein the into-the-Sun versus out-of-the-Sun polarity of the solar-wind magnetic field changes. These magnetic sector reversals typically occur prior to intervals of high-speed wind.

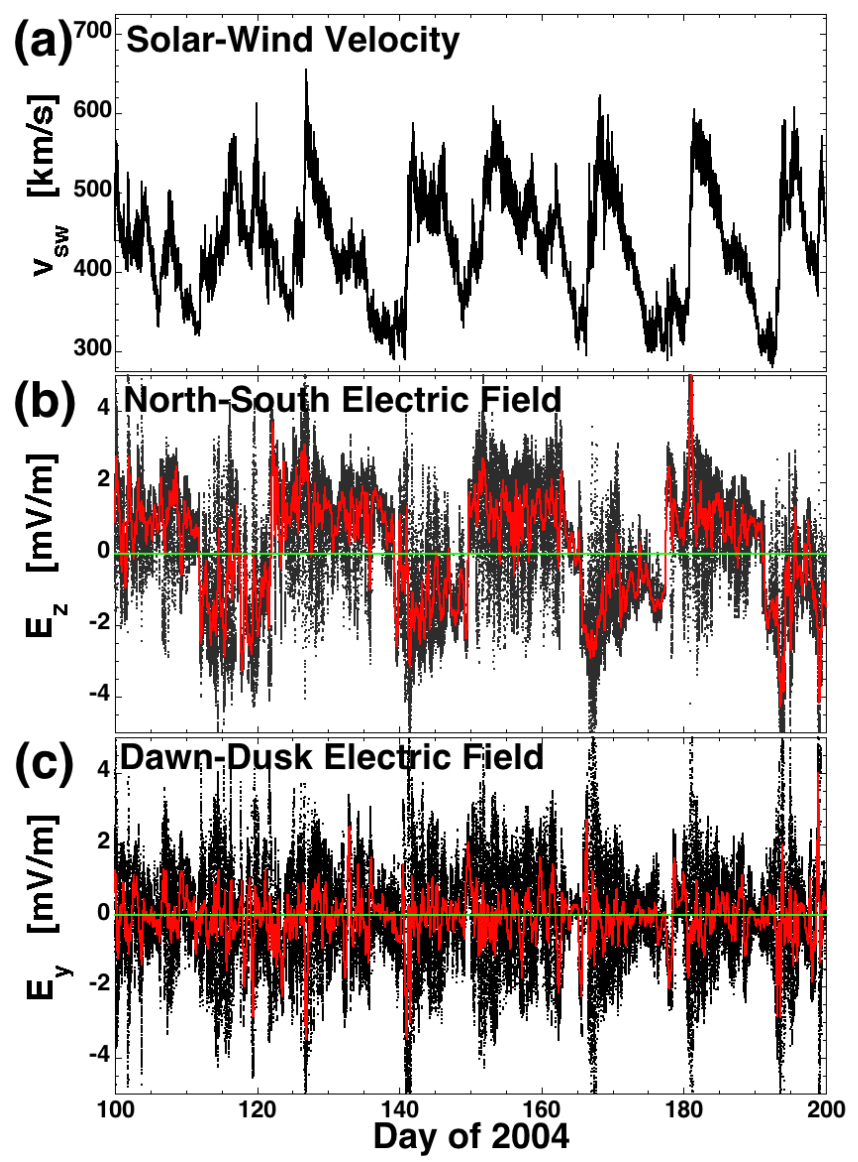

Figure 1. 100 days of the motional electric field $\underline{E}$ from the ACE spacecraft upstream of the Earth are shown. In the top panel the solar-wind speed $v_{s w}$ is plotted with 64-s time resolution. In the middle panel the north-south component of $\underline{E}$ is plotted: black points are 64-s time resolution and the red points are 24-hr running averages of the black points. In the bottom panel the dawn-dusk component of $\underline{E}$ is plotted: black points are 64-s time resolution and the red points are 24-hr running averages of the black points. The GSE coordinate system is used. 
A higher-time-resolution look at the motional electric field of the solar wind appears in Figure 2. Here, plasma [18] and magnetic-field [19] measurements from the WIND spacecraft with 3-s time resolution are used to calculate $\underline{E}=-(1 / c) \underline{v}_{s w} \times \underline{B}$. In the top panel $E_{y}$ and $E_{z}$ are plotted for two hours of fast solar wind and in the bottom panel $E_{y}$ and $E_{z}$ are plotted for two hours of slow solar wind. Note in Figure 2 that the temporal behavior of the motional electric field is dominated by sudden changes of the values of $E_{y}$ and $E_{z}$; these sudden changes occur when a current sheet in the solar wind passes the spacecraft. This is the "mesoscale structure" of the solar wind, dominated by magnetic flux tubes bounded by current sheets.

In the upper two panels of Figure 3 the vector tips of the solar-wind motional electric field $\underline{E}=-(1 / c) \underline{v}_{s w} \times \underline{B}$ are plotted in the $\mathrm{E}_{\mathrm{y}}$-versus- $\mathrm{E}_{\mathrm{z}}$ plane. The left panel is for 24-hr averages of the electric field (from the multispacecraft OMNI2 data set [20]) and the right panel is for 64-s values of the electric field (from the ACE spacecraft). The 24-hr averages are much more dominated by a north-south orientation to $\underline{E}$, whereas the 64 -s values in the right panel are more isotropic. Basically, the deviation of $\underline{E}=-(1 / c) \underline{v}_{s w} \times \underline{B}$ from purely north-south is owed to the deviation of the solar-wind magnetic field $\underline{B}$ from the Parker-spiral direction. In the lower two panels of Figure 3 the vector tips of the solar-wind magnetic field $\underline{B}$ are plotted in the $B_{y}$-versus- $B_{z}$ plane, left panel being 24-hr averages of the magnetic field (from OMNI2) and the right panel being 64-s values (from ACE). The 24-hr averages of the electric and magnetic field (left panels) are dominated by a Parker-spiral orientation with $B_{y}>0$ and $E_{z}>0$ for out-of-the-Sun (away) magnetic fields and $B_{y}<0$ and $E_{z}<0$ for into-the-Sun (toward) magnetic fields.
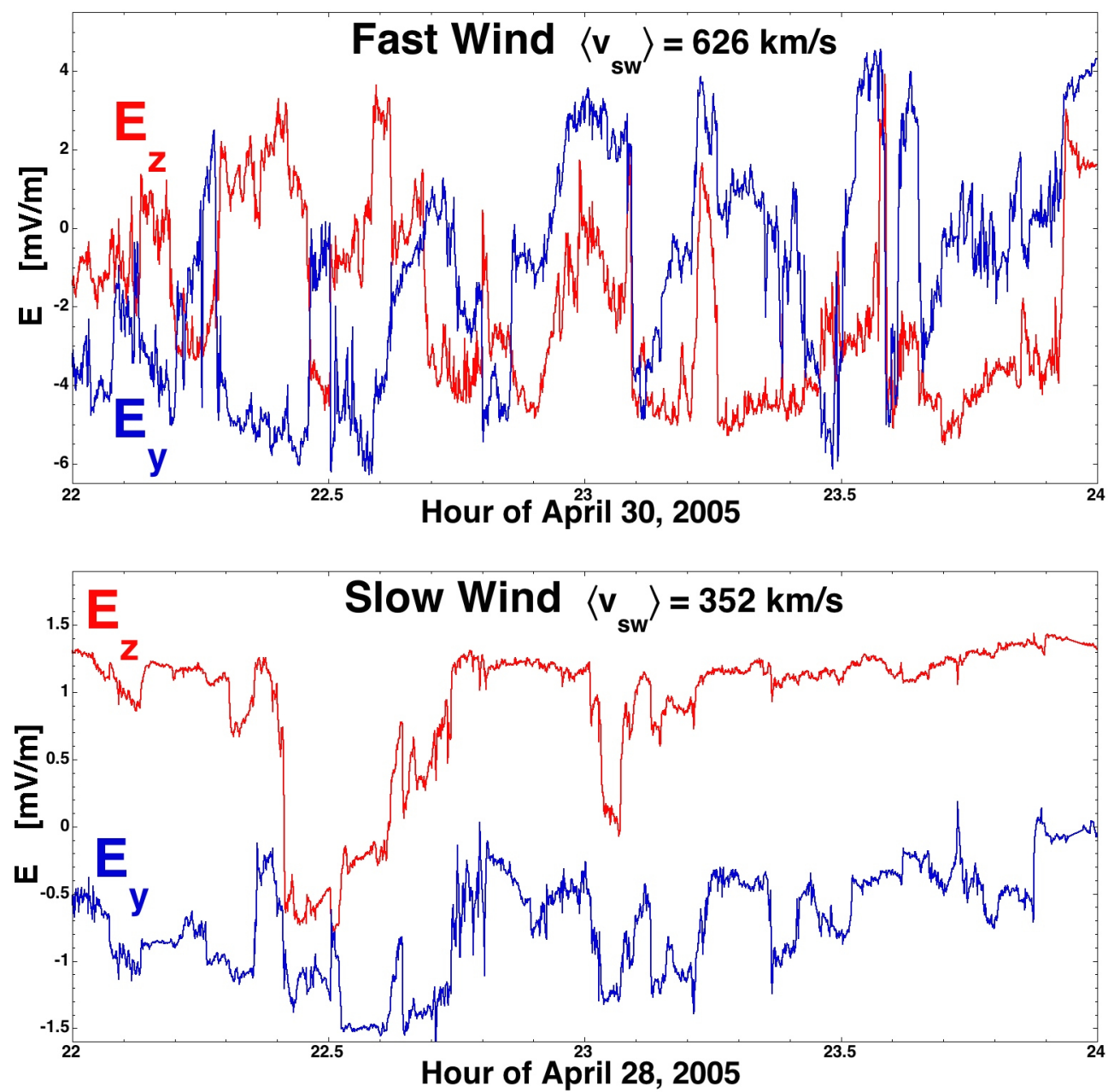

Figure 2. The motional electric field of the solar wind is plotted for 2 hours of fast solar wind (top panel) and for 2 hours of slow solar wind (bottom panel). The 3-s time-resolution values are from the WIND spacecraft upstream of the Earth. $E_{y}$ is in red and $E_{z}$ is in blue. The GSE coordinate system is used. 

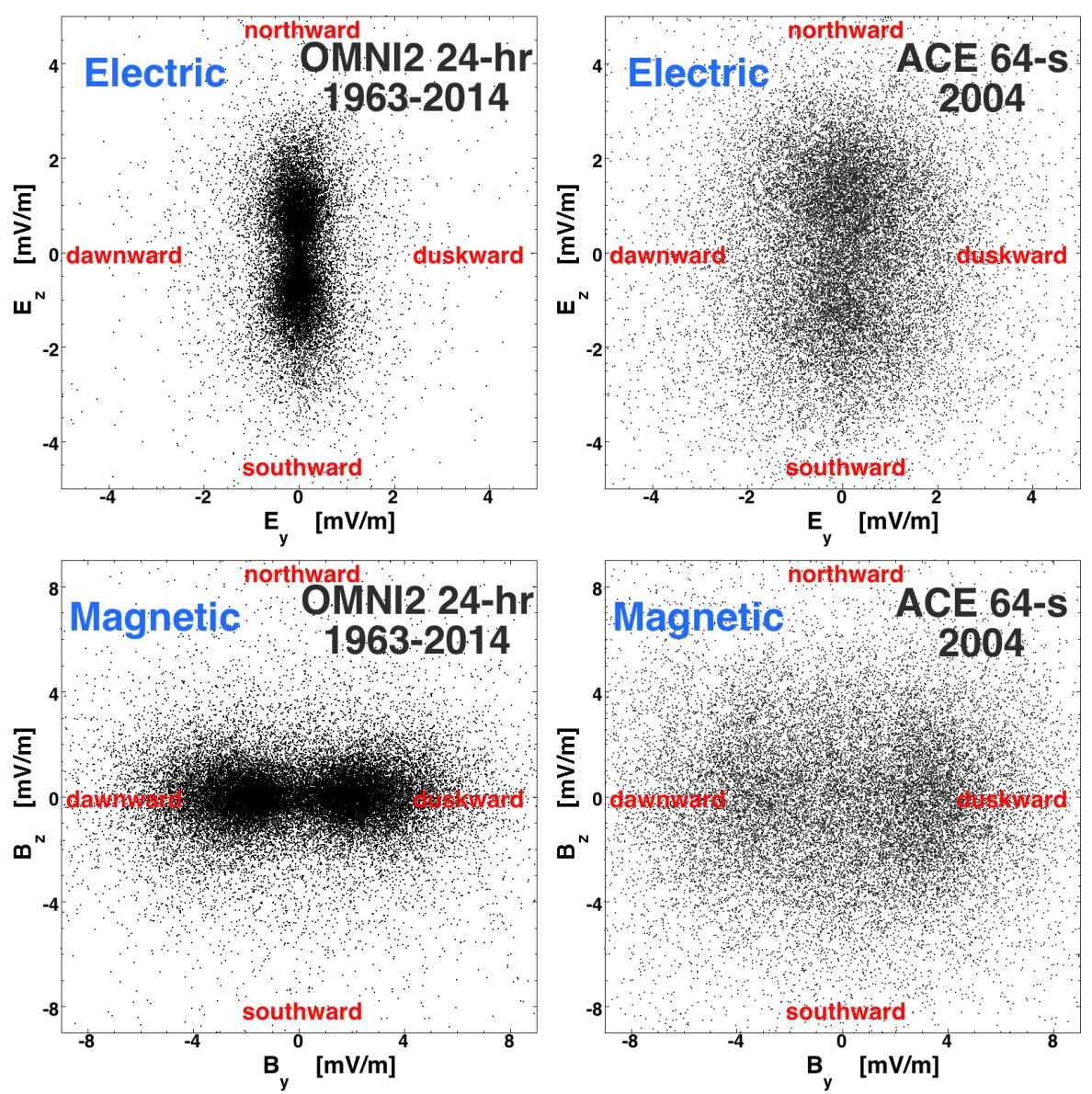

Figure 3. In the top two panels the vector tips of solar-wind motional electric field at Earth are plotted in the (GSE) $E_{z}$-versus- $E_{z}$ plane. The plane is viewed from the Sun looking at the Earth. In the left panel 24-hr averages of $\underline{E}$ from OMNI2 are used and in the right panel 64-s resolution values of $E$ from ACE are used. In the bottom two panels the vector tips of the solar-wind magnetic field are plotted, 24-hr averages from OMNI2 on the left and 64-s values from ACE on the right.

\section{The Parker-Spiral Motional Electric Field}

Owing to the 27-day rotation of the Sun, the magnetic field in the solar-wind plasma has a global Archimidesspiral pattern. In $(r, \theta, \phi)$ spherical coordinates the Parker-spiral magnetic field $\underline{B}$ at the radius $\mathrm{r}$ from the Sun and colatitude $\theta$ in the heliosphere is given by [1]

$$
\underline{B}=\left(B_{o} r_{o}^{2} r^{-2}, 0,-B_{o} \omega r_{o}^{2} r^{-1} v_{s w}^{-1} \sin \theta\right)
$$

where $B_{o}$ is a reference magnetic-field strength near the Sun (with $B_{o}>0$ for an out-of-the-Sun (away) field and $B_{o}<0$ for an into-the-Sun (toward) field); $r_{o}$ is the reference radius where $B_{o}$ is measured; $\omega \approx 2.9 \times 10^{-6}$ radians/s is the angular rotation rate of the solar surface at the solar equator; and $v_{s w}$ is the solar-wind radial velocity. The angle $\theta=0^{\circ}$ at the north pole of the Sun and $\theta=180^{\circ}$ at the south pole. In general, the Parker-spiral magnetic field of the heliosphere is of one sector (away or toward) in the northern hemisphere of the heliosphere and is of the opposite sector (toward or away) in the southern hemisphere, with a heliospheric current sheet separating the two morphologies [21]. In Figure 4 the Parker-spiral motional electric field of the solar wind will be sketched with the heliospheric current sheet lying in the equatorial plane bounding an away sector $\left(B_{o}>0\right)$ in the northern hemisphere and a toward sector $\left(B_{o}<0\right)$ in the southern hemisphere.

For the Parker-spiral magnetic field given by expression (7), the motional electric field of expression (2) for a radial solar-wind velocity $\underline{v}_{s w}=\left(v_{s w}, 0,0\right)$ is

$$
\underline{E}=\left(0, E_{\theta}, 0\right)
$$




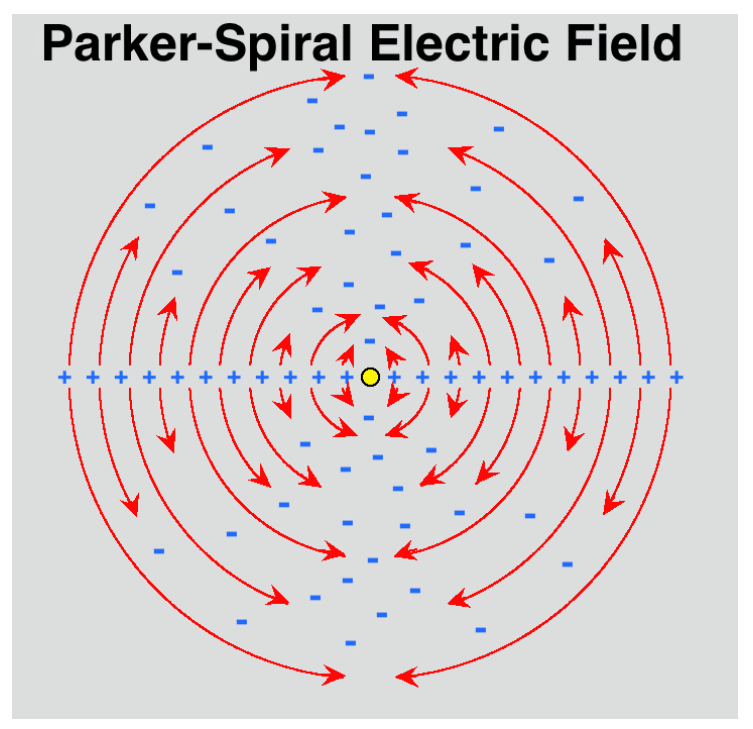

Figure 4. As viewed from an observer in the ecliptic plane far from the Sun (yellow circle), Parker-spiral electric field lines are sketched in red and the charge density supporting that electric field is sketched in blue. North is toward the top. In this sketch the Parker-spiral magnetic field is away from the Sun in the northern hemisphere and toward the Sun in the southern hemisphere. The positively charged heliospheric current sheet is seen horizontally across the center of the sketch.

$$
E_{\theta}=-\left(B_{o} \omega r_{o}^{2} / c\right) r^{-1} \sin \theta
$$

this electric field is sketched as the red field lines in Figure 4. The motional electric field $\underline{E}$ for the Parker spiral is purely poloidal: $\underline{E}$ is northward for an away-from-the-Sun magnetic-field orientation and $\underline{E}$ is southward for a toward-the-Sun magnetic-field orientation. Note in expression (8) that $E_{\theta}$ does not depend on $v_{s w}$.

Examining expression (8) in spherical coordinates one finds $\nabla \times \underline{E}=0$ everywhere for the Parker-spiral electric field. The distributed charge density in the solar-wind plasma that supports this global electrostatic electric field is given by Coulomb's law $\nabla \cdot \underline{E}=4 \pi \rho_{q}$ : taking the divergence of expression (8) in spherical coordinates yields

$$
\rho_{q}=-\left(B_{o} \omega r_{o}^{2} / 2 \pi c\right) r^{-2} \cos \theta
$$

a distributed charge density throughout the heliosphere. This charge density is sketched as the blue "+" and "-c" symbols in Figure 4. According to expression (9) the charge density is strong near the polar regions of the heliosphere $\left(\theta \sim 0^{\circ}\right.$ and $\left.\theta \sim 180^{\circ}\right)$ and weak near the equatorial regions $\left(\theta \sim 90^{\circ}\right)$. For an away-from-the-Sun magnetic-field orientation $\left(B_{o}>0\right)$ the distributed charge density $\rho_{q}$ is negative in the northern hemisphere and positive in the southern hemisphere and for a toward orientation $\left(B_{o}<0\right)$ the distributed charge density $\rho_{q}$ is positive in the northern hemisphere and negative in the southern hemisphere. Note in expression (9) that $\rho_{q}$ does not depend on the solar-wind velocity $\mathrm{v}_{\mathrm{sw}}$.

With $\nabla \times \underline{E}=0$ everywhere, the global Parker-spiral electric field is purely electrostatic with its origin in regions where $\nabla \cdot \underline{E} \neq 0$. Note in Figure 4 that the electric-field lines of the global Parker-spiral motional electric field end.

The distributed current density $j$ in the solar-wind plasma that supports the global Parker-spiral magnetic-field geometry is calculated by applying Ampere's law $\nabla \times \underline{B}=(4 \pi / c) j$ to the Parker-spiral magnetic field given by expression (7): taking the curl of expression (7) in spherical coordinates yields

$$
\underline{j}=\left(j_{r}, 0,0\right)
$$




$$
j_{r}=-\left(B_{o} c \omega r_{o}^{2} / 2 \pi\right) v_{s w}^{-1} r^{-2} \cos \theta .
$$

This distributed current density is purely radial and it is strong in the polar regions of the heliosphere and weak in the equatorial regions, with $j_{r}$ negative (toward the Sun) when the magnetic field is away from the Sun $\left(B_{o}>0\right)$ and $j_{r}$ is positive (away from the Sun) when the magnetic field is toward the Sun $\left(B_{o}<0\right)$.

Taking the heliospheric current sheet to lie in the equatorial plane of the heliosphere there is a radial component of the current in the sheet associated with the jump in the azimuthal component of the Parker-spiral magnetic field across the current sheet and there is an azimuthal current in the sheet associated with the reversal of the radial component of the magnetic field across the current sheet. Taking the thickness of the heliospheric current sheet to be $d$, Ampere's law gives

$$
\begin{gathered}
j_{r} d=(c / 4 \pi) \Delta B_{\phi}=\left(c B_{o} \omega r_{o}^{2} / 2 \pi\right) v_{s w}^{-1} r^{-1} \\
j_{\phi} d=(c / 4 \pi) \Delta B_{r}=\left(c B_{o} r_{o}^{2} / 2 \pi\right) r^{-2},
\end{gathered}
$$

for the magnitudes of the radial and azimuthal current densities in the heliospheric current sheet, note that the thickness $d$ of the heliospheric current sheet could be a function of $r$, so the current density in the heliospheric current sheet could vary with $r$ differently than the right-hand sides of expressions (11a) and (11b).

Note that the poloidal motional electric field of the solar wind reverses across the current sheet owing to the reversal of the direction of $\underline{B}$ across the sheet as $v_{s w}$ remains radially outward. This electric-field reversal across the current sheet is sketched in Figure 4. There is a nonzero charge density in the equatorial current sheet associated with the reversal of the poloidal electric field: Coulomb's law gives this charge density $\rho_{q}$ as

$$
\rho_{q} d=(1 / 4 \pi) \Delta E_{\theta}=\left(B_{o} \omega r_{o}^{2} / 2 \pi c\right) r^{-1}
$$

where again $d$ is the thickness of the current sheet, and where again $d$ could be a function of $r$. The charge density of the heliospheric current sheet is positive if the magnetic field is outward (from the Sun) in the northward hemisphere and inward in the southern hemisphere (as drawn in Figure 4): the charge density of the heliospheric current sheet is negative if the magnetic field is inward in the northern hemisphere and outward in the southern hemisphere. The source of this charge density is the relativistic transformation of current density into charge density [22], which will be discussed in Section 5.1.

Owing to the orbital motion of the Earth around the Sun, the radial component $B_{r}$ of the Parker-spiral magnetic field gives rise to an additional north-south poloidal electric field as seen by the Earth. The orbital velocity of the Earth around the Sun is $v_{\text {orbit }} \approx 29.8 \mathrm{~km} / \mathrm{s}$ in the dawnward direction, which is small compared with $v_{s w}$ and so the orbital motional electric field is small compared with the Parker-spiral motional electric field. The motional electric field $E_{\text {orbit }}=(1 / c) v_{\text {orbit }} B_{r}$ in the reference frame of the Earth is southward when the Earth is in an away magnetic sector and northward when the Earth is in a toward magnetic sector, always opposite to the sign of the Parker-spiral motional electric field.

In the real solar wind there are several complications to this ideal-Parker-spiral picture. First, the global solar wind contains solar ejecta, which are impulsively ejected large-scale plasmas with non-Parker-spiral-oriented magnetic fields. Second, the solar-wind velocity is not uniform everywhere: rather there are regions on the rotating Sun that emit fast wind and regions that emit slower wind, disturbing the uniform-Parker-spiral picture with compressions and rarefactions. Third, there is a tilt to the solar magnetic dipole, plus magnetic multipoles that invalidate the uniform-northern-hemisphere uniform-southern-hemisphere picture of Figure 4. Fifth, the heliospheric current sheet was drawn to be planar in Figure 4 but in reality it is tilted and warped. Finally, there are large-amplitude fluctuations in the magnetic-field orientation about the Parker-spiral direction representing a mesoscale spatial structure of the solar-wind magnetic field. This mesoscale structure is considered in the following section.

\section{The Mesoscale Electric Field Structure of the Solar Wind}

In this section, the mesoscale spatial structure of the solar-wind motional electric field in the reference frame of the Earth is examined. That electric-field spatial structure is associated with the mesoscale magnetic-field spatial structure of the solar-wind plasma, which is largely flux tubes separated by current sheets [3] [4]. The advection of these magnetic spatial structures past the Earth results in temporal variations of $\underline{E}$ and $\underline{B}$ at the Earth mani- 
fested as sudden large changes in the vectors $\underline{E}$ and $\underline{B}$ associated with each current sheet passing the Earth (cf. Figure 2).

\subsection{Idealized Cases}

The question of whether the mesoscale solar-wind electric field is an electrostatic field originating from charge density or an induction field originating from time-varying current density is first examined in two idealized cases drawn in the ecliptic plane in Figure 5. The first case (top panel) has the current sheets of the solar wind

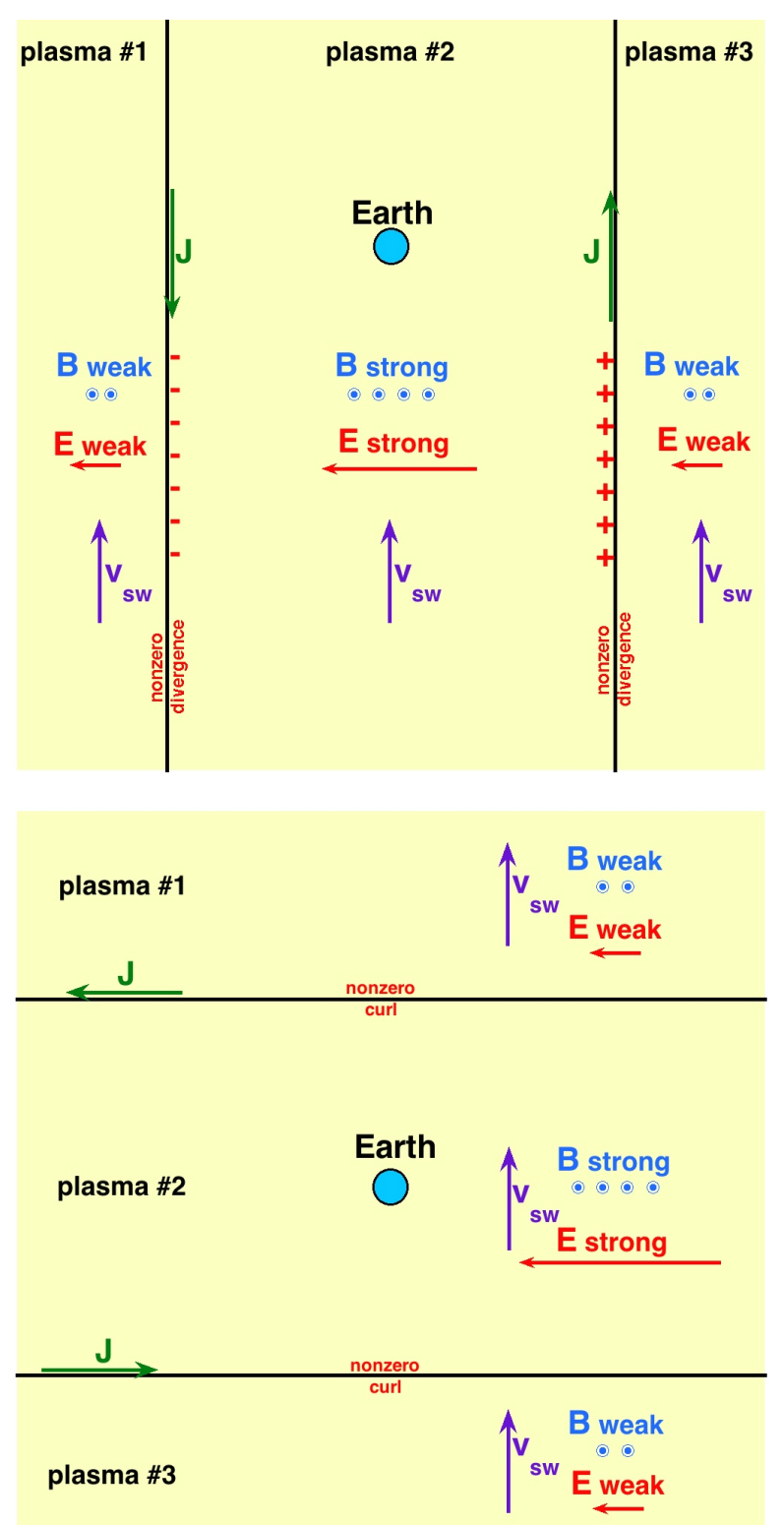

Figure 5. Two idealized sketches in the ecliptic plane of the solar wind plasma advecting past the Earth with current sheets of infinite extent. In the top panel the current sheets of the solar wind (black) are oriented parallel to the solar wind velocity vector and in the bottom panel the current sheets of the solar wind are oriented with their normal parallel to the solar wind velocity vector. The direction of the solar-wind velocity is indicated in purple, the direction of the magnetic field is indicated in blue, and the direction of the motional electric field (in the Earth's frame) is indicated in red. The motional electric field in the top panel is purely electrostatic (divergence origin, curl-free) and the motional electric field in the bottom panel is purely inductive (curl origin, divergence-free). 
oriented along the Sun-Earth line and the second case (bottom panel) has the current sheets of the solar wind oriented normal to the Sun-Earth line. In both cases the plasma in the vicinity of the Earth is divided by two current sheets (black lines) into three plasmas, with the Earth within plasma \#2. In both cases the solar-wind plasma is taken to have the uniform velocity $\underline{v}_{s w}$ (purple vector) everywhere, with $\underline{v}_{s w}$ parallel to the Sun-Earth line, with the Sun off the bottom of the drawing in the distance. In both cases plasma \#2 is taken to have a strong uniform magnetic field $\underline{B}$ and plasmas $\# 1$ and $\# 3$ are taken to have weaker uniform magnetic fields $\underline{B}$; the $\underline{B}$ vector (blue) is taken everywhere to be vertical out of the plane of the sketches with the strength of $\underline{B}$ changing across the current sheets.

In the top panel of Figure 5 the direction of the current density $j$ in each current sheet is indicated by a green arrow, with the jump in the magnetic-field vector $\underline{B} \underline{B}$ across the current sheet being related to the current density. In the reference frame of the Earth, the motional electric field in the solar wind is given by $\underline{E}=-(1 / c) \underline{v}_{s w} \times \underline{B}$ in all three plasmas with the same value of $\underline{\mathrm{v}}_{\mathrm{sw}}$ : the motional electric field $\underline{E}=-(1 / c) \underline{v} \times \underline{B}$ (red vectors) is uniform and strong within plasma $\# 2$ and $\underline{E}=-(1 / c) \underline{v} \times \underline{B}$ is uniform and weak in plasmas \#1 and \#3. In the top panel of Figure 5 the normal component of the motional electric field changes across the current sheets, which is of the nature of $\nabla \cdot \underline{E} \neq 0$ in the sheets; hence according to expression (2) there are nonzero charge densities $\rho_{q}$ in the current sheets of the top panel. These charge densities are indicated by the red "-" and "+" symbols in the sketch. This charge density may seem at first paradoxical to the reader because in the reference frame of the solar-wind plasma (where $\underline{\mathrm{v}}=0$ everywhere and $\underline{E}=0$ everywhere) there is $\nabla \cdot \underline{E}=0$ and no $\rho_{q}$ at the current sheets. The nonzero $\rho_{q}$ seen in the Earth's reference frame has its origin as a Lorentz transformation

$$
\rho_{q}=\left(1 / c^{2}\right) \underline{v} \cdot \underline{j}
$$

of the 3 -vector current density $j$ in a current density 4 -vector $\left(j, c \rho_{q}\right)$ [22] [23]. (Of course the total charge is conserved in the Lorentz transformation, but the spatial volumes containing the ions and the electrons moving at different velocities in the nonzero current are Lorentz contracted differently, resulting in a nonzero charge density out of equal amounts of positive and negative charge [14] [23].) As can be seen by examining the geometry of the electric field drawn in the top panel of Figure 5, there is no variation in $\underline{E}$ in the direction perpendicular to $\underline{E}$, so $\nabla \times \underline{E}=0$. Because $\nabla \times \underline{E}=0$, in the top panel of Figure 5 the motional electric field of the mesoscale structure of the solar wind given by expression (2) as seen by the Earth is purely electrostatic $\underline{E}_{e s}$ and has its origin in regions of nonzero charge density where the divergence of the electric field is nonzero.

As an aside, note that if one applies the Lorentz transformation of expression (13) to the radial current density $\mathrm{j}_{\mathrm{r}}$ that supports the Parker-spiral magnetic field (expression (10)), then one gets the distributed charged density $\rho_{q}$ (expression (9)) of the Parker-spiral electric field. Likewise, a Lorentz transformation of the current density in the heliospheric current sheet that corresponds to the reversal of the Parker-spiral magnetic field (expression (11a)) yields the charge density of the heliospheric current sheet that corresponds to the reversal of the Parker-spiral electric field (expression (12)). Again, those charge densities arise from a difference in the Lorentz contraction of the volumes containing the differently moving ions and electrons in the currents.

The second idealized case (bottom panel of Figure 5) has the current sheets of the solar wind oriented with the current-sheet normal vectors parallel to the solar-wind velocity vector $\underline{v}_{s w}$. In the sketch two current sheets (black) divide the region into three plasmas, with the magnetic-field strength of plasma $\# 2$ uniform and strong and the field strength in plasmas \#1 and \#3 uniform and weak. The velocity $\underline{v}_{s w}$ (purple vector) is everywhere the same and so the motional electric field $\underline{E}=-(1 / c) \underline{v} \times \underline{B}$ (red vector) as seen by the Earth is uniform and strong in plasma \#2 and uniform and weak in each of plasmas \#1 and \#3. In the bottom panel of Figure 5 the tangential component of the motional electric field $\underline{E}$ changes across the current sheets, which is of the nature of $\nabla \times \underline{E} \neq 0$, with a nonzero curl. At the locations of the current sheets there is a nonzero $\partial j / \partial t$ in the reference frame of the Earth owing to the motion of the current sheet as seen by the Earth. Hence, in the bottom panel of Figure 5 the motional electric field of the solar wind given by expression (2) as seen by the Earth is purely inductive $\underline{E}_{i n d}$ and has its origin at regions of $\partial j / \partial t$ (e.g. $\partial \underline{B} / \partial t$ ) where the curl of the electric field is nonzero.

\subsection{A Parker-Spiral-Aligned Flux Tube}

The constructions in the two panels of Figure 5 were idealizations to produce a purely electrostatic motional electric field (top panel) and a purely induction motional electric field (bottom panel). In actuality the current sheets of the solar wind have a wide range of orientations [2] [4] [24], and the current sheets are not infinite in 
extent [2] [24]. Usually, the magnetic field of the solar wind at Earth is approximately in the Parker-spiral orientation with the individual magnetic-flux tubes aligned approximately with the Parker-spiral direction [25]. In the top panel of Figure 6 a single Parker-spiral-oriented magnetic flux tube (pink) is sketched in the ecliptic plane at Earth. The magnetic field orientation (blue vectors) is away from the Sun inside the flux tube and the motional electric field in the reference frame of the Earth (red vectors) is northward directed up out of the ecliptic plane. The magnetic-field strength is sketched strong within the tube and weak outside of the tube so the motional electric field is strong within the tube and weak outside of the tube. In the bottom panel of Figure 6 the Parker-spiral-oriented flux tube of the top panel is viewed along the axis of the tube looking back along the spiral toward the Sun with the magnetic field pointed at the viewer. The up-down direction in the sketch of the bottom panel is the Earth's north-south direction and the flux tube is drawn to have a square cross section. The direction of the current density in the current sheets (black) surrounding the pink flux tube is indicated with green vectors. In the reference frame with the Earth at rest, the tube and its surrounding plasma advects toward the right with the solar-wind velocity $\underline{v}_{s w}$ away from the Sun; the projection of $\underline{v}_{s w}$ is drawn as the purple vector in the bottom panel. The motional electric field $\underline{E}=-(1 / c) \underline{v} \times \underline{B}$ in the reference frame of the Earth is indicated as the northward-pointing red vectors, strong within the pink flux tube and weak within the yellow surrounding plasma. The nature of the electric-field change across the top and bottom current sheets in the sketch is $\nabla \cdot \underline{E} \neq 0$ and there is a nonzero charge density $\rho_{q}$ in those top and bottom current sheets (which have
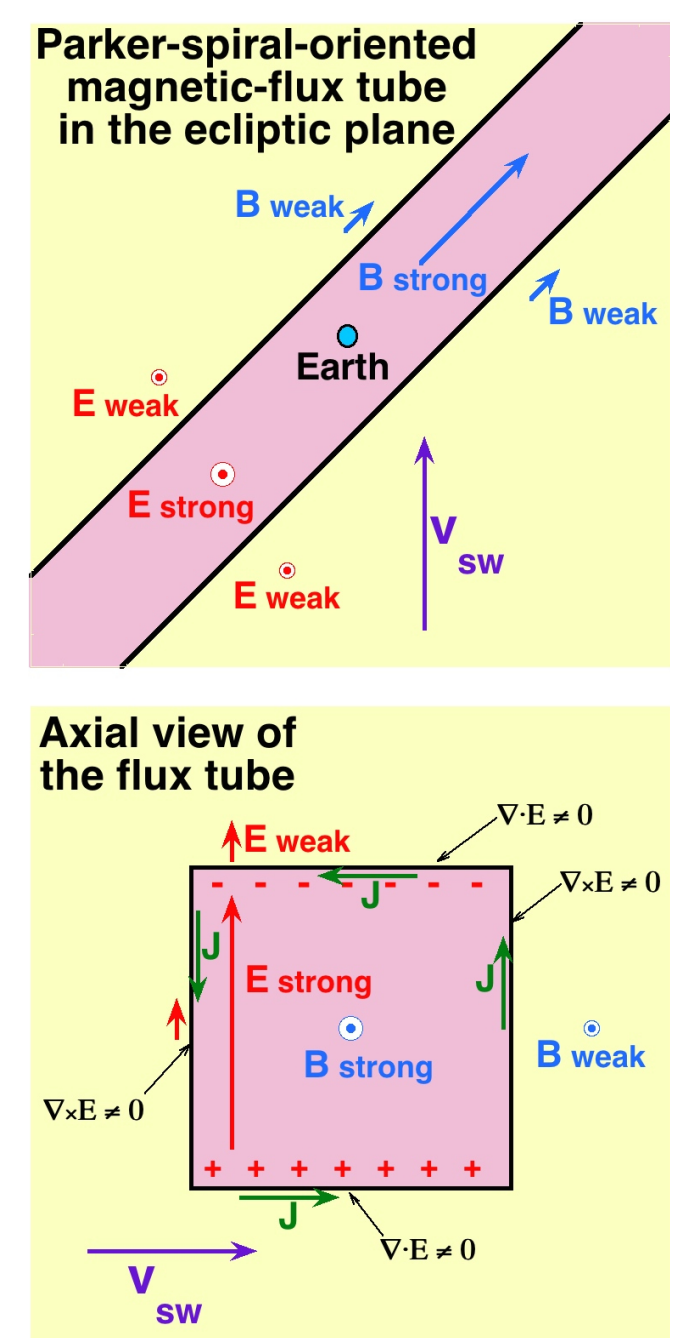

Figure 6. In the top panel a Parker-spiral-oriented flux tube passing the Earth is sketched in the ecliptic plane; the magnetic field in the tube has an "away from the Sun" orientation. In the bottom panel the same flux tube is viewed looking down the axis of the tube back toward the Sun. In both panels the electric fields are sketched in the reference frame of the Earth. 
$\underline{v}_{s w} \cdot \underline{j} \neq 0$ ). The nature of the electric-field change across the leading (right) and trailing (left) current sheets in the sketch is $\nabla \times \underline{E} \neq 0$ and, owing to the motion of the current sheets, there is a nonzero $\partial j / \partial t$ in those leading and trailing current sheets (which have current-sheet normals $\underline{n}$ oriented with $\underline{n} \cdot \underline{v}_{s w} \neq 0$ ). The top and bottom current sheets make a contribution to an electrostatic electric field in the tube and the leading and trailing current sheets make a contribution to an induction electric field in the tube.

In Figure 7, the Earth's-reference-frame charge density $\rho_{q}=\left(1 / c^{2}\right) \underline{v}_{s w} \cdot \underline{j}$ and the Earth's-reference-frame $\partial \underline{B} / \partial t=-\left(\underline{v}_{s w} \cdot \nabla\right) \underline{B}$ on the current sheets of a square flux tube are numerically integrated to calculate the divergence-origin electric field $\underline{E}_{e s}$ (top panel) and the curl-origin electric field $\underline{E}_{\text {ind }}$ (middle panel) in and around the flux tube:
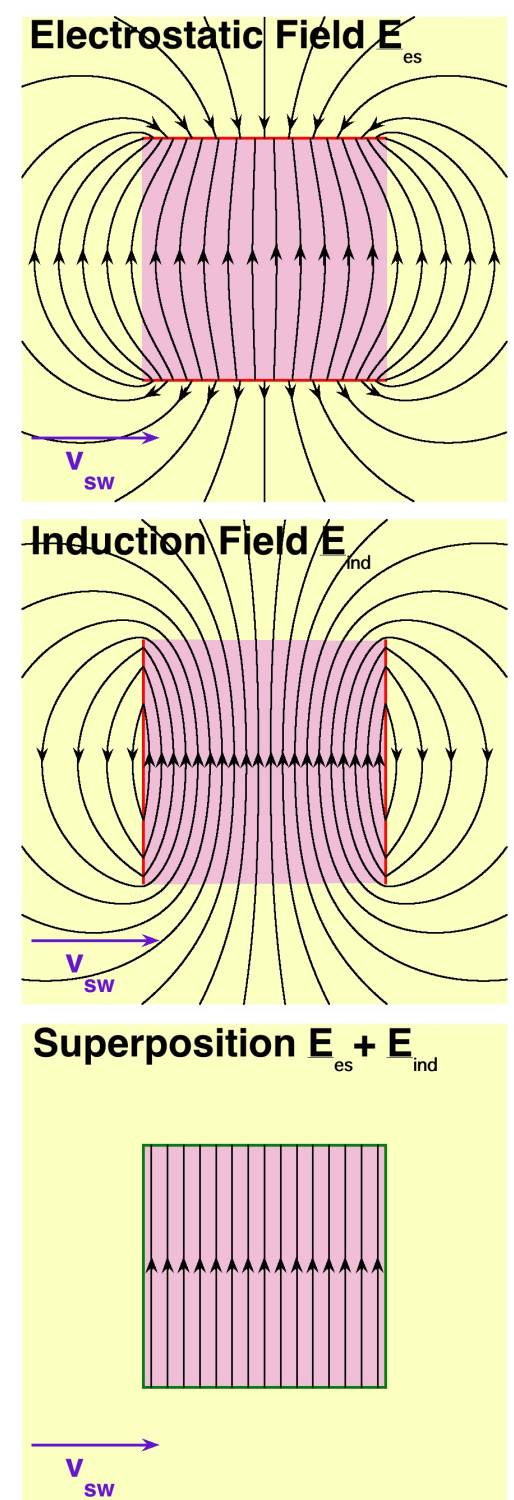

Figure 7. For the flux tube drawn in the bottom panel of Figure 6 (with the magnetic field pointing up out of the plane of the figure) the electric fields in the Earth's reference frame are numerically calculated. In the top panel the $\nabla \cdot \underline{E}=4 \pi \rho_{q}$ electrostatic electric field is calculated everywhere from the Coulomb's integral over the charge density in the top and bottom current sheets, in the middle panel the $\nabla \times \underline{E}=-(1 / c) \partial \underline{B} / \partial t$ induction electric field is calculated everywhere from the BiotSavart integral over the $\partial \underline{B} / \partial t$ in the left and right current sheets, and in the bottom panel the superposition of the two fields is shown. 


$$
\begin{gathered}
\nabla \cdot \underline{E}_{e s}=\left(4 \pi / c^{2}\right) \underline{v}_{s w} \cdot \underline{j} \\
\nabla \times \underline{E}_{\text {ind }}=(1 / c)\left(\underline{v}_{s w} \cdot \nabla\right) \underline{B}
\end{gathered}
$$

The superposition $\underline{E}=\underline{E}_{e s}+\underline{E}_{\text {ind }}$ of the two electric fields is calculated in the bottom panel: this superposed electric field $\underline{E}$ is the field that would be measured by a detector. In Figure 7 the tube is taken to have a uniform magnetic field $B$ within the tube and zero magnetic field outside the tube. The current density $j$ in each of the four current sheets bounding the tube is given by Ampere's law to be $j w=(c / 4 \pi) B$, where $\mathrm{w}$ is the thickness of a current sheet. As is the case for the flux tube in the bottom panel of Figure 6, the flux tube in Figure 7 is taken to advect in the Earth's frame from left to right with the solar-wind velocity and the magnetic field inside the tube points out of the page of the sketch. The charge density in the top and bottom current sheets is given by the relativistic transformation (taking $\gamma=1$ ) $\rho_{q}=\left(1 / c^{2}\right) \underline{v}_{s w} \cdot j$ (expression (13)), so the charge density is $\rho_{q} w=-(1 / 4 \pi c) v_{s w} B$ in the top current sheet and $\rho_{q} w=+(1 / 4 \pi c) v_{s w} B$ in the bottom sheet. The $\partial \underline{B} / \partial t=-\left(\underline{v}_{s w} \cdot \nabla\right) \underline{B}$ in the leading and trailing current sheets of the tube are taken to have $\partial \underline{B} / \partial t=\Delta \underline{B} / \Delta t= \pm B w v_{s w}$, with $\partial \underline{B} / \partial t$ positive (up out of the page of the figure) in the leading edge and negative (into the page) in the trailing edge. For the top and bottom current sheets of the flux tube the electrostatic electric field $\underline{E}_{e s}$ everywhere in space is numerically calculated from expression (14a) by the Coulomb integral over the charge densities of the top and bottom sheets (e.g. eq. (5.15) of [6])

$$
\underline{E}_{e s}(\underline{x})=\int \rho_{q}\left(\underline{x}^{\prime}\right)\left(\underline{x}-\underline{x}^{\prime}\right)\left|\underline{x}-\underline{x}^{\prime}\right|^{-3} \mathrm{~d}^{3} x^{\prime} .
$$

A sampling of electric-field lines $\underline{E}_{e s}(\underline{x})$ are numerically traced in the top panel of Figure 7. Note that these electrostatic-electric-field lines end. For the right and left current sheets of the flux tube the induction electric field $\underline{E}_{\text {ind }}$ everywhere in space is numerically calculated from the Biot-Savart integrals applied to Faraday's law of induction (expression (14b)) with the integration over $\partial \underline{B} / \partial t$ in the right and left current sheets (e.g. eq. (5.14) of [6])

$$
\underline{E}_{\text {ind }}(\underline{x})=-(1 / 4 \pi c) \int\left\{\partial \underline{B}\left(\underline{x}^{\prime}\right) / \partial t \times\left(\underline{x}-\underline{x}^{\prime}\right)\right\}\left|\underline{x}-\underline{x}^{\prime}\right|^{-3} \mathrm{~d}^{3} x^{\prime} .
$$

A sampling of the induction electric-field lines are numerically traced and plotted in the second panel of Figure 7. Note that these induction-electric-field lines do not end. The vector sum (superposition) of the electrostatic field $\underline{E}_{e s}$ and the induction field $\underline{E}_{\text {ind }}$ is numerically calculated everywhere in space and a sampling of the field lines of this superposed field are plotted in the third panel of Figure 7. Note that the superposition $\underline{E}_{e s}+\underline{E}_{i n d}$ of the electrostatic field and the induction field is a uniform electric field within the flux tube and zero electric field outside of the flux tube (cf. expression (2)). Note also in the bottom panel of Figure 7 that the electric field lines begin and end on the top boundaries (where there is nonzero charge density) and that the tangential component of the electric field jumps across the left and right moving boundaries (where there is nonzero $\partial j / \partial t$ ).

In the top panel of Figure 8 the ratio $R=\left(\left|\underline{E}_{e s}\right|-\left|\underline{E}_{\text {ind }}\right|\right) /\left(\left|\underline{E}_{e s}\right|-\left|\underline{E}_{\text {ind }}\right|\right)$ formed from the magnitudes of the electrostatic field $\underline{E}_{e s}$ and the induction field $\underline{E}_{\text {ind }}$ is calculated at every point inside the square flux tube of Figure 7. The ratio $\mathrm{R}$ is +1 if the electric field is purely electrostatic and $R$ is -1 of the electric field is purely inductive. In Figure 8 colors approaching $R=+1$ are red and colors approaching $R=-1$ are violet. As can be seen in the top panel of Figure 8, the field has an electrostatic dominance near the top and bottom of the tube (where the charge density resides) and the field has an induction dominance near the leading and trailing edges (where the $\partial \underline{B} / \partial t$ or $\partial j / \partial t$ reside). The green curve in Figure 9 is a binning of the value of $R$ for all points inside the square flux tube of the top panel of Figure 8; the mean value and standard deviation of the $R$ values in the interior of the square flux tube is $\langle R>=0.0 \pm 0.14$.

In the middle and bottom panels of Figure 8 the ratio $R=\left(\left|\underline{E}_{e s}\right|-\left|\underline{E}_{\text {ind }}\right|\right) /\left(\left|\underline{E}_{e s}\right|+\left|\underline{E}_{\text {ind }}\right|\right)$ is plotted in color for a flux tube with a cross section that is tall in the north-south direction (middle) and a flux tube with a cross section that is elongated in the sunward-antisunward direction (bottom). Red colors represent regions where the value of $R$ approaches +1 (electrostatic dominance) and violet colors represent regions where the value of $R$ approaches -1 (induction dominance). The tall flux tube in the middle panel has a motional electric field that is dominantly induction, with the mean (and standard deviation) value $\langle R>=-0.53 \pm 0.19$ for the interior of the tube. The flattened flux tube in the bottom panel has a motional electric field that is dominantly electrostatic, with the mean (and standard deviation) value $\langle R>=+0.53 \pm 0.19$ for the interior of the tube. In Figure 9 the 

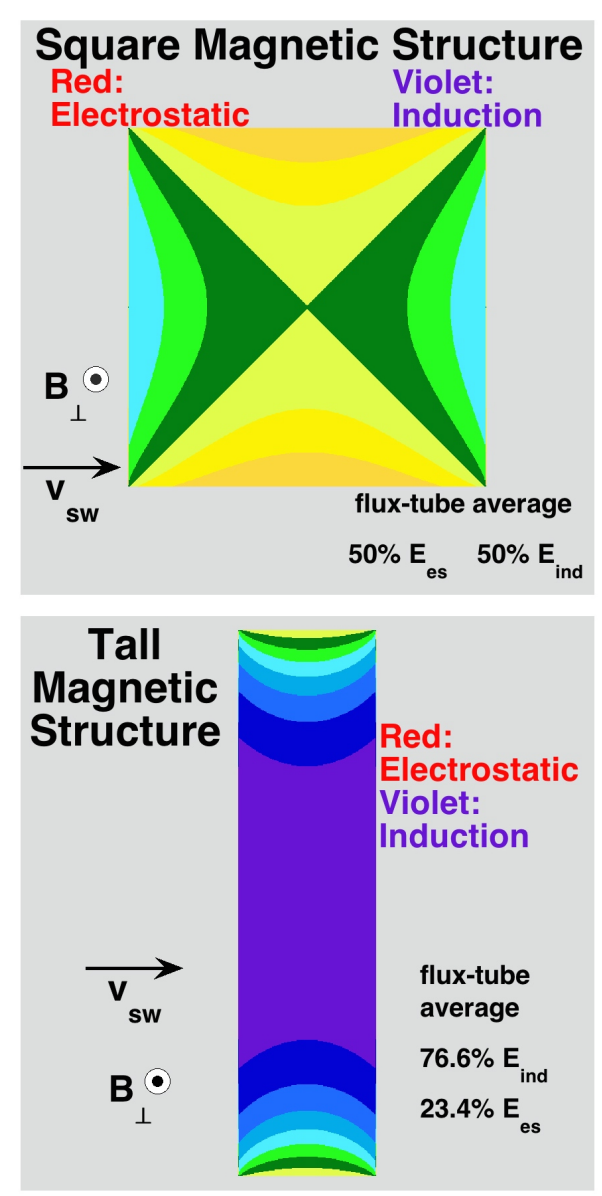

\section{Flat Magnetic Structure}

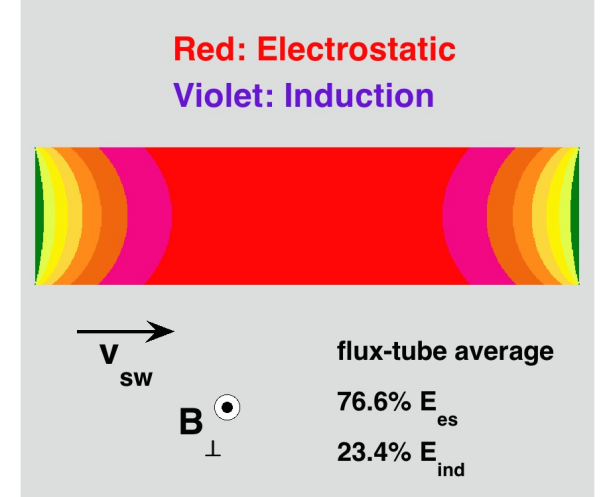

Figure 8. For flux tubes similar to the one in Figure 7, the local value of the ratio $R=\left(\left|\underline{E}_{e s}\right|-\left|\underline{E}_{\text {ind }}\right|\right) /\left(\left|\underline{E}_{e s}\right|+\left|\underline{E}_{\text {ind }}\right|\right)$ is plotted in color, with red colors representing values near +1 (electrostatic dominating) and purple colors representing values near -1 (induction dominating). In the top panel the cross section of the flux tube is square, in the middle panel the cross-section of the flux tube is tall with a 4-to-1 height-to-width ratio, and in the bottom panel the cross section of the flux tube is squat with a 1-to-4 height-to width ratio.

local values of $\mathrm{R}$ are binning for all points inside the tall flux tube of the middle panel of Figure 8 (violet curve) and for all points inside the flattened flux tube of the bottom panel of Figure 8 (red curve); inside of the tall flux tube the induction electric field dominates almost everywhere and inside of the flattened flux tube the electrostatic electric field dominates almost everywhere. 


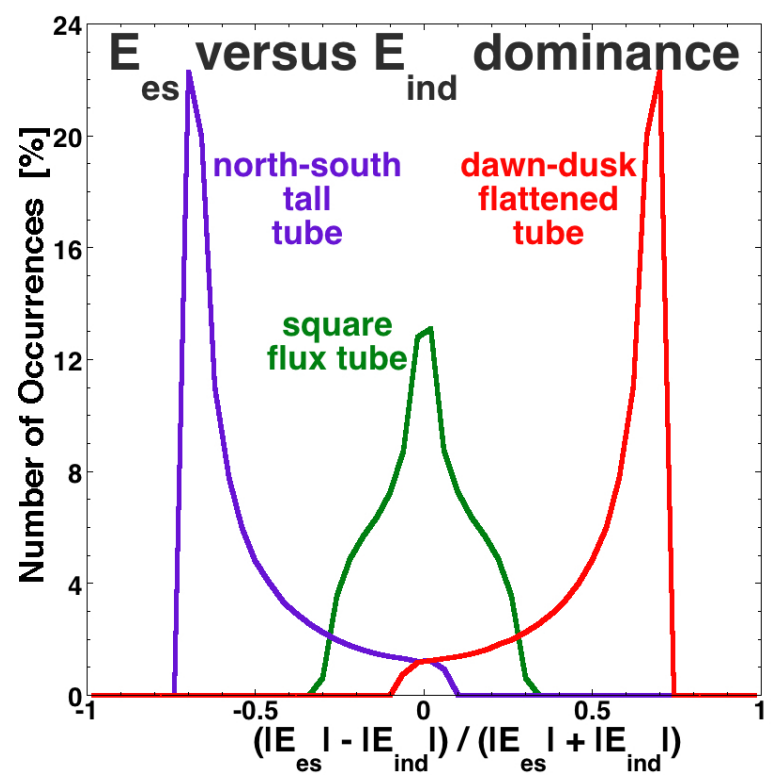

Figure 9. For the three flux tubes of Figure 8, the local value of $\left(\left|\underline{E}_{e s}\right|-\left|\underline{E}_{\text {ind }}\right|\right) /\left(\left|\underline{E}_{e s}\right|+\left|\underline{E}_{\text {ind }}\right|\right)$ is binned for the entirety of each flux tube.

\subsection{The Mesoscale Structure of the Solar Wind}

In reality the mesoscale magnetic structure of the solar-wind plasma is quite complicated, resembling a "spaghetti" of tangled tubes of various cross-sectional shapes, various cross-sectional sizes, and various orientations [3] [4]. Instead of the idealized rectangular cross sections utilized in Section 5.2, the cross-sectional shapes of solar-wind flux tubes are more likely a Voronoi pattern of tubes packed against each other, each with a complicated polygonal shape (cf. Figure 1 of [4]). Portions of current sheets that are obliquely aligned with the solar-wind velocity vector will have both $\rho_{q}=\left(1 / c^{2}\right) \underline{v}_{s w} \cdot \underline{j}$ and $\partial \underline{B} / \partial t=-\left(\underline{v}_{s w} \cdot \nabla\right) \underline{B}$ (i.e. $\nabla \cdot \underline{E} \neq 0$ and $\nabla \times E \neq 0$ ), giving rise to electrostatic field and to induction field. Examining the panels of Figure 8, it is seen that if the cross-sectional shape of a flux tube is tall in the north-south direction, then the leading-edge and trailing-edge current sheets will dominate and the electric field within the tube will have dominantly an induction $\partial j / \partial t$ source; on the contrary if the cross-sectional shape of a flux tube is flat in the ecliptic plane then the top and bottom current sheets will dominate and the electric field within the tube will have dominantly an electrostatic $\rho_{q}$ source. In general, at Earth the electrostatic versus induction dominance of the time variations of the solar-wind motional electric field will vary with time as magnetic structures with varying shapes pass the Earth. However, regions of the solar wind that are compressed or rarefacted show systematic changes to the measured current-sheet orientations indicating flux tubes that are flattened in the radial direction (in compression regions) or stretched in the radial direction (in rarefaction regions) [26]. Therefore, in regions where the solar-wind plasma has undergone large-scale radial compressions (e.g. behind interplanetary shocks or in corotating interaction regions) the solar-wind motional electric field of the mesoscale structure should be dominantly induction and in regions where the solar-wind plasma has undergone large-scale radial rarefactions (e.g. in the trailing edges of high-speed streams and behind fast magnetic clouds) the solar-wind motional electric field of the mesoscale structure should be dominantly electrostatic.

\section{Summary, Refinements, and Conclusion}

A Helmholtz decomposition of the motional electric field in the solar wind was made and the electrostatic versus induction nature of the field was analyzed as was the charge-density origin versus the time-varying-currentdensity origin of the field. To calculate the motional electric fields, Lorentz transformations were used; when Lorentz transforming the electric and magnetic fields, the currents and charges also had to be Lorentz transformed. Just as the transformation of the motional electric field $\underline{E}$ from $\underline{B}$ is important at non-relativistic so- 
lar-wind velocities, the transformation of the charge density $\rho_{q}$ from the current density $j$ is important at non-relativistic velocities.

The large-scale time-averaged electric field of the solar wind associated with the outward advection of the Parker-spiral magnetic field in the solar-wind plasma is an electrostatic electric field having its source in the distributed charge density in the solar-wind plasma and the more-concentrated charge density of the heliospheric current sheet. This large-scale electric field is in the poloidal direction, northward at Earth in away magnetic sectors and southward at Earth in toward magnetic sectors. The origin of the large-scale electric field is a relativistic transformation of the large-scale magnetic field in the solar-wind plasma and the origin of the distributed charge density is the same relativistic transformation of the distributed current density in the solar-wind plasma that supports the Parker-spiral magnetic field.

The mesoscale structure of the solar-wind electric field spatially varies from being dominantly electrostatic to being dominantly inductive. At the Earth the time-varying electric field will be dominantly inductive near current sheets that have their normal vectors nearly aligned with the Sun-Earth line and the time-varying electric field will be dominantly electrostatic near current sheets that have their normal vectors nearly perpendicular to the Sun-Earth line. In general the mesoscale electric field will be a combination of electrostatic and induction electric fields, varying with time as differently shaped magnetic structures pass over the Earth. In compression regions of the solar wind time-varying induction fields will tend to dominate, and in rarefaction regions of the solar wind time-varying electrostatic fields will tend to dominate.

Note that in the actual solar wind the solar-wind velocity vector $\underline{v}_{s w}$ also has spatial structure, on both large scales and mesoscales. On the mesoscale, there is usually a jump in the plasma velocity vector $\underline{v}$ as well as the magnetic-field vector $\underline{B}$ across the current sheets in the solar wind [27]. The jump in $\underline{v}$ across the current sheet amounts to a vorticity $\underline{\omega}=\nabla \times \underline{v}$ in the sheets; in a plasma there is a charge density $\rho_{q}$ associated with vorticity [28] with the charge density in a vorticity layer given by eq. (11) of [9]

$$
\rho_{q}=-(1 / 4 \pi c) \underline{\omega} \cdot \underline{B} .
$$

(Note that for charge density associated with vorticity there is no reference frame in which the charge density is zero.) In general the change in velocity $\underline{\underline{v}}$ across a solar-wind current sheet is much smaller in magnitude than the bulk flow velocity $\underline{v}_{s w}$ of the solar-wind plasma, so the inhomogeneity of the flow-velocity field is typically a less-than- $10 \%$ effect on magnitude of the motional electric fields of the solar wind.

To conclude, a first-principles understanding of the motional electric field of the solar wind associated with the advection of magnetic structure in the solar-wind plasma has been developed. This understanding is based on the Lorentz transformations of electric and magnetic fields, the Lorentz transformations of current densities and charge densities, and the application of Maxwell's equations.

\section{Discussion: Charge Density in the Solar-Wind Plasma}

As seen in Sections 4 and 5, there is a nonzero charge density $\rho_{q}$ in the solar-wind plasma associated with the motional electric field of the solar-wind: a distributed charge density associated with the advection of the global Parker-spiral magnetic field and more-concentrated charge densities in the current sheets of the solar wind. The physical origin of that charge density is the difference in the Lorentz contractions of volumes containing the positive ions of the solar wind and the negative electrons of the solar wind when the ions and electrons move at (slightly) different velocities owing to the presence of an electrical current. The differences in ion versus electron velocity are on the order of $1 \mathrm{~km} / \mathrm{s}$ in the solar-wind current sheets.

The charge density in the solar-wind plasma associated with the motional electric field is frame dependent, being zero in the reference frame of the plasma and nonzero in other reference frames such as the frame of the Earth or the Sun.

In the directions perpendicular to $\underline{B}$, the solar-wind plasma behaves on large scales as an ideal-MHD fluid: in the parallel-to- $\underline{B}$ direction the MHD description of the collisionless solar wind can fail (cf. Sect. 1 of [29] for MHD tests that are failed by the solar wind). Sometimes in space physics textbooks it is declared that MHD has $\nabla \cdot \underline{E}=0$ (e.g. eq. (5.22) of Parks [30], eq. (4.82) of Gombosi [31], eq. (6.1.31) of Gurnett and Bhattacharjee [32], or Table 6-6 of Knipp [33]) or equivalently textbooks declare that $\rho_{q}=0$ (e.g. eq. (5.23) of Parks [30] or Sect. 6.1 of Gurnett and Bhattacharjee [32]), which cannot be true relativistically (cf. expressions (13) and (17)) and which is not true in MHD computer simulations of the solar-wind flow (cf. Sect. 2.3 of reference [9]). 
In deriving ideal MHD, a quasineutrality condition $n_{p} \approx n_{e}$ (ion number density approximately equals the electron number density) is often assumed. The assumption of quasineutrality does not mean that Coulomb's law $\nabla \cdot \underline{E}=4 \pi \rho_{q}$ is irrelevant or inconsistent with the application of ideal MHD.

For the charge density in the solar wind, one can assess just how quasineutral the solar-wind plasma is by calculating the fractional charge imbalance $n_{q} / n_{o}$, where $n_{q}=\rho_{q} / e$ is the number density of excess charges and where $n_{o} \approx n_{p} \approx n_{e}$ is the number density of the plasma. A current sheet of the mesoscale structure of the solar wind plasma is considered. If the magnetic-field vector jumps by $\Delta B$ across the current sheet of thickness $w$, then the current density in the sheet is $j=c \Delta B / 4 \pi w$. The charge density $\rho_{q}$ in the current sheet from the transformation $\rho_{q}=\left(1 / c^{2}\right) \underline{v} \cdot j$ is then $\rho_{q}=v_{s w} \Delta B / 4 \pi c w$ and $n_{q}=\rho_{q} / e=v_{s w} \Delta B / 4 \pi c e w$. If the magnetic-field vector across the current sheet jumps by $\Delta B=5 \times 10^{-5}$ Gauss, if the width of the current sheet is $w=2500 \mathrm{~km}$, and if the speed of the solar wind is $v_{s w}=438 \mathrm{~km} / \mathrm{s}$ (cf. Table 1), then the estimate for the number density of charges is $n_{q}=4.8 \times 10^{-8} \mathrm{~cm}^{-3}$. A typical number density of the solar-wind plasma is $n_{o}=6.8 \mathrm{~cm}^{-3}$ (cf. Table 1 ). Thus $n_{q} / n_{o} \sim 10^{-8}$ for the charge density of the solar wind.

This amount of charge imbalance is small, but it is not zero. Even with $n_{q} / n_{o} \sim 10^{-8}$ in the solar wind, the limit $\rho_{q}=0$ cannot be taken and $\nabla \cdot \underline{E} \neq 0$ : this is demonstrated with the following example. One can compare the solar-wind degree of quasineutrality to the degree of quasineutrality in a lightning bolt. The linear charge density $\mathrm{Q} / \mathrm{L}$ in a conducting lightning channel is about $\mathrm{Q} / \mathrm{L}=10^{-4} \mathrm{C} / \mathrm{m}$ [34]. Dividing by e, this is a number of excess charges per unit length $\mathrm{N} / \mathrm{L}=6.2 \times 10^{6} \mathrm{~cm}^{-1}$. A conducting lightning channel has a radius $\mathrm{r}$ of about $r=1 \mathrm{~cm}$ [35], so the net number density of charges $n_{q}$ in the channel is $n_{q}=2 \times 10^{6} \mathrm{~cm}^{-3}$. The number density $n_{o}$ of free electrons in the highly ionized lightning channel is about $n_{o}=10^{18} \mathrm{~cm}^{-3}$ [36]. Hence, the fractional charge density in the channel is $n_{q} / n_{o} \sim 2 \times 10^{-12}$. (In actuality, the charge density $n_{q}$ inside the channel is lower than $2 \times$ $10^{6} \mathrm{~cm}^{-3}$ since the strong radial electrostatic electric field of the charged lightning channel pushes excess charge out of the channel where it migrates radially outward into the unionized air, into a "corona sheath" of radius of a meter or so [37]). Note that the lightning channel, with an electrostatic potential of $\sim 10^{8} \mathrm{~V}$ [34], is several orders of magnitude more charge neutral than the $n_{q} / n_{o} \sim 10^{-8}$ of the solar-wind plasma. Lightning is an electrodynamic problem in which Coulomb's law (Gauss' law, Poisson's equation, capacitance calculations) cannot be ignored (e.g. [38] [39]).

Further, the condition of quasineutrality does not mean that the electric field is negligibly small. Certainly in calculating test-particle orbits in a plasma (or MHD fluid) both $\underline{E}$ and $\underline{B}$ are needed [40]. In Gaussian-cgs units the electric field $E$ and the magnetic field $B$ have the same units $\left(1\right.$ gauss $=1$ Stat $\left.V o l t / c m=1 \mathrm{gm}^{1 / 2} \mathrm{~cm}^{-1 / 2} \mathrm{~s}^{-1}\right)$ so their magnitudes are directly comparable: even though the magnitude of the motional electric field is $v / c$ times smaller than the magnitude of the magnetic field (cf. expression (2)), the electric force on a particle $q \underline{E}$ is $c / v$ times larger than the magnetic force $q(1 / c) \underline{v} \times \underline{B}$ on a particle.

\section{Acknowledgements}

The author wishes to thank Joachim Birn, Mick Denton, Raluca Ilie, and Dan Welling for useful conversations. All spacecraft data utilized are freely available from NASA data servers http://cdaweb.gsfc.nasa.gov and http://omniweb.gsfc.nasa.gov. This work was supported at the Space Science Institute by the NSF Solar-Terrestrial Program via grant AGS-1261659, by the NASA Heliophysics LWS TRT program via grants NNX14AN90G and NNX16AB75G, and by the NASA Heliophysics Guest Investigator Program via grant NNX14AC15G and at the University of Michigan by the NASA Geospace SR\&T Program via grant NNX12AD29G.

\section{References}

[1] Parker, E.N. (1965) Dynamical Theory of the Solar Wind. Space Science Reviews, 4, 666-708. http://dx.doi.org/10.1007/BF00216273

[2] Weimer, D.R., Ober, D.M., Maynard, N.C., Burke, W.J., Collier, M.R., McComas, D.J., Ness, N.F. and Smith, C.W. (2002) Variable Time Delays in the Propagation of the Interplanetary Magnetic Field. Journal of Geophysical Research: Space Physics, 107, 1210. http://dx.doi.org/10.1029/2001JA009102

[3] Bruno, R., Carbone, V., Veltri, P., Pietropaolo, E. and Bavassano, B. (2001) Identifying Intermittency Events in the Solar Wind. Planetary and Space Science, 49, 1201-1210. http://dx.doi.org/10.1016/S0032-0633(01)00061-7

[4] Borovsky, J.E. (2008) The Flux-Tube Texture of the Solar Wind: Strands of the Magnetic Carpet at 1 AU? Journal of Geophysical Research: Space Physics, 113, A08110. http://dx.doi.org/10.1029/2007ja012684 
[5] Alfven, H. and Falthammar, K.G. (1963) Cosmical Electrodynamics. 2nd Edition, Sect. 1.3, Oxford University Press, Oxford.

[6] Jackson, J.D. (1975) Classical Electrodynamics. 2nd Edition, Sects. 6.4, 6.5, 10.2, 11.10, and 12.4, Wiley, New York.

[7] Landau, L.D. and Lifshitz, E.M. (1960) Electrodynamics of Continuous Media. Sect. 49, Pergamon Press, New York.

[8] Goertz, C.K., Shan, L.-H. and Smith, R.A. (1993) Prediction of Geomagnetic Activity. Journal of Geophysical Research: Space Physics, 98, 7673-7684. http://dx.doi.org/10.1029/92JA01193

[9] Borovsky, J.E. and Birn, J. (2014) The Solar-Wind Electric Field Does Not Control the Dayside Reconnection Rate. Journal of Geophysical Research: Space Physics, 119, 751-760. http://dx.doi.org/10.1002/2013JA019193

[10] Zaharia, S., Jordanova, V.K., Thomsen, M.F. and Reeves, G.D. (2008) Self-Consistent Geomagnetic Storm Simulation: The Role of Induced Electric Fields. Journal of Atmospheric and Solar-Terrestrial Physics, 70, 511-518. http://dx.doi.org/10.1016/j.jastp.2007.08.067

[11] Ilie, R., Daldorff, L.K.S. and Liemohn, M.W. (2015) Calculating the Inductive Electric Field in the Terrestrial Magnetosphere. Journal of Geophysical Research, 2015JA022034R.

[12] Lemaire, J. and Scherer, M. (1971) Kinetic Models of the Solar Wind. Journal of Geophysical Research, 76, 74797490. http://dx.doi.org/10.1029/JA076i031p07479

[13] Griffiths, D.J. (1999) Introduction to Electrodynamics. 3rd Edition, Sect. 10.3 and Appendix B, Prentice Hall, Upper Saddle River.

[14] Kusse, B.R. and Westwig, E.A. (2006) Mathematical Physics. 2nd Edition, Sect. 2.5.4, Wiley-VCH, Weinheim.

[15] Noerdlinger, P.D. (1971) Boundary Conditions for Moving Magnetic Fields and Lorentz Transformation of Surface Currents. American Journal of Physics, 39, 191-192. http://dx.doi.org/10.1119/1.1986090

[16] McComas, D.J., Blame, S.J., Barker, P., Feldman, W.C., Phillips, J.L., Riley, P. and Griffee, J.W. (1998) Solar Wind Electron Proton Alpha Monitor (SWEPAM) for the Advanced Composition Explorer. Space Science Reviews, 86, 563612. http://dx.doi.org/10.1023/A:1005040232597

[17] Smith, C.W., Acuna, M.H., Burlaga, L.F., L'Heureux, J., Ness, N.F. and Scheifele, J. (1998) The ACE Magnetic Fields Experiment. Space Science Reviews, 86, 613-632. http://dx.doi.org/10.1023/A:1005092216668

[18] Lin, R.P., Anderson, K.A., Ashford, S., Carlson, C., Curtis, D., Ergun, R., Larson, D., McFadden, J., McCarthy, M., Parks, G.K., Reme, H., Bosqued, J.M., Coutelier, J., Cotin, F., D’uston, C., Wenzel, K.-P., Sanderson, T.R., Henrion, J., Ronnet, J.C. and Paschmann, G. (1995) A Three-Dimensional Plasma and Energetic Particle Investigation for the WIND Spacecraft. Space Science Reviews, 71, 125-153. http://dx.doi.org/10.1007/BF00751328

[19] Lepping, R.P., Acuna, M.H., Burlaga, L.F., Farrell, W.M., Slavin, J.A., Schatten, K.H., Mariani, F., Ness, N.F., Neubauer, F.M., Whang, Y.C., Byrnes, J.B., Kennon, R.S., Panetta, P.V., Scheifele, J. and Worley, E.M. (1995) The WIND Magnetic Field Investigation. Space Science Reviews, 71, 207-229. http://dx.doi.org/10.1007/BF00751330

[20] King, J.H. and Papitashvili, N.E. (2005) Solar Wind Spatial Scales in and Comparisons of Hourly Wind and ACE Plasma and Magnetic Field Data. Journal of Geophysical Research: Space Physics, 110, A02104. http://dx.doi.org/10.1029/2004JA010649

[21] Hundhausen, A.J. (1995) The Solar Wind. In: Kivelson, M.G. and Russell, C.T., Eds., Introduction to Space Physics, Sect. 4.4, Cambridge University Press, New York, 91-128.

[22] Podolsky, B. (1947) On the Lorentz Transformation of Charge and Current Densities. Physical Review, 72, $624-626$. http://dx.doi.org/10.1103/PhysRev.72.624

[23] Gabuzda, D.C. (1987) Magnetic Force Due to a Current-Carrying Wire: A Paradox and Its Resolution. American Journal of Physics, 55, 420-422. http://dx.doi.org/10.1119/1.15124

[24] Malaspina, D.M. and Gosling, J.T. (2012) Two Spacecraft Observations of Magnetic Discontinuities in the Solar Wind with STEREO. Journal of Geophysical Research: Space Physics, 117, A04109. http://dx.doi.org/10.1029/2011ja017375

[25] Borovsky, J.E. (2010) On the Variations of the Solar-Wind Magnetic Field about the Parker-Spiral Direction. Journal of Geophysical Research: Space Physics, 115, A09101. http://dx.doi.org/10.1029/2009ja015040

[26] Borovsky, J.E. and Denton, M.H. (2016) The Trailing Edges of High-Speed Streams at 1 AU. Journal of Geophysical Research: Space Physics, 121, 6107-6140. http://dx.doi.org/10.1002/2016ja022863

[27] Borovsky, J.E. (2012) The Effect of Sudden Wind Shear on the Earth's Magnetosphere: Statistics of Wind-Shear Events and CCMC Simulations of Magnetotail Disconnections. Journal of Geophysical Research: Space Physics, 117, A06224. http://dx.doi.org/10.1029/2012ja017623

[28] Borovsky, J.E. and Hansen, P.J. (1998) The Morphological Evolution and Internal Convection of E $\times$ B-Drifting Plas- 
ma Clouds: Theory, Dielectric-in-Cell Simulations, and N-Body Dielectric Simulations. Physics of Plasmas, 5, $3195-$ 3223. http://dx.doi.org/10.1063/1.872988

[29] Borovsky, J.E. and Gary, S.P. (2009) On Viscosity and the Reynolds Number of MHD Turbulence in Collisionless Plasmas: Coulomb Collisions, Landau Damping, and Bohm Diffusion. Physics of Plasmas, 16, 082307. http://dx.doi.org/10.1063/1.3155134

[30] Parks, G.K. (1991) Physics of Space Plasmas. Sect. 5.4.4, Addison-Wesley, New York.

[31] Gombosi, T.I. (1998) Physics of the Space Environment. Sects. 4.4 and 14.4.4, Cambridge University Press, Cambridge. http://dx.doi.org/10.1017/CBO9780511529474

[32] Gurnett, D.A. and Bhattacharjee, A. (2005) Introduction to Plasma Physics with Space and Laboratory Applications. Sect. 6.1.3, Cambridge University Press, Cambridge.

[33] Knipp, D.J. (2011) Understanding Space Weather and the Physics behind It. McGraw-Hill Learning Solutions, Boston.

[34] Borovsky, J.E. (1998) Lightning Energetics: Estimates of Energy Dissipation in Channels, Channel Radii, and Channel-Heating Risetimes. Journal of Geophysical Research: Atmospheres, 103, 11537-11553. http://dx.doi.org/10.1029/97JD03230

[35] Jones, R.C. (1968) Return Stroke Core Diameter. Journal of Geophysical Research, 73, 809-814. http://dx.doi.org/10.1029/JB073i002p00809

[36] Ripoll, J.-F., Zinn, J., Colestock, P.L. and Jeffrey, C.A. (2014) On the Dynamics of Hot Air Plasmas Related to Lightning Discharges: 2. Electrodynamics. Journal of Geophysical Research: Atmospheres, 119, 9218-9235. http://dx.doi.org/10.1002/2013jd020068

[37] Maslowski, G. and Rakov, V.A. (2006) A Study of the Lightning Channel Corona Sheath. Journal of Geophysical Research: Atmospheres, 111, D14110. http://dx.doi.org/10.1029/2005jd006858

[38] Borovsky, J.E. (1995) An Electrodynamic Description of Lightning Return Strokes and Dart Leaders: Guided-Wave Propagation along Conducting Cylindrical Channels. Journal of Geophysical Research: Atmospheres, 100, 2697-2726. http://dx.doi.org/10.1029/94jd00407

[39] Dwyer, J.R. and Uman, M.A. (2014) The Physics of Lightning. Physics Reports, 534, 147-241. http://dx.doi.org/10.1016/j.physrep.2013.09.004

[40] Birn, J., Thomsen, M.F., Borovsky, J.E., Reeves, G.D., McComas, D.J., Bellian, R.D. and Hesse, M. (1997) Substorm Ion Injections: Geosynchronous Observations and Test Particle Orbits in Three-Dimensional Dynamic MHD Fields. Journal of Geophysical Research: Space Physics, 102, 2325-2341. http://dx.doi.org/10.1029/96ja03032

\section{Submit or recommend next manuscript to SCIRP and we will provide best service for you:}

Accepting pre-submission inquiries through Email, Facebook, LinkedIn, Twitter, etc.

A wide selection of journals (inclusive of 9 subjects, more than 200 journals)

Providing 24-hour high-quality service

User-friendly online submission system

Fair and swift peer-review system

Efficient typesetting and proofreading procedure

Display of the result of downloads and visits, as well as the number of cited articles

Maximum dissemination of your research work

Submit your manuscript at: http://papersubmission.scirp.org/ 